\title{
Combined and simultaneous endoscopic endonasal and transorbital surgery for a Meckel's cave schwannoma: technical nuances of a mini-invasive, multiportal approach
}

\author{
Alberto Di Somma, MD, PhD, FEBNS, ${ }^{1}$ Cristobal Langdon, MD, ${ }^{2}$ Matteo de Notaris, MD, PhD, ${ }^{3}$ \\ Luis Reyes, MD, ${ }^{1}$ Santiago Ortiz-Perez, MD, PhD, ${ }^{4,5}$ Isam Alobid, MD, PhD, ${ }^{3}$ \\ and Joaquim Enseñat, MD, PhD' \\ Departments of ${ }^{1}$ Neurological Surgery and ${ }^{2}$ Otorhinolaryngology, Hospital Clínic de Barcelona, Spain; ${ }^{3}$ Department of \\ Neuroscience, "G. Rummo" Hospital, Benevento, Italy; ${ }^{4}$ Department of Ophthalmology, Hospital Clínic de Barcelona; and \\ ${ }^{5}$ Department of Ophthalmology, Hospital Virgen de las Nieves, Granada, Spain
}

OBJECTIVE Over the years, Meckel's cave pathologies have been judged off-limits because of high rates of morbidity. Even though several studies have defined various surgical routes with tolerable morbidity and mortality rates, controversies related to the optimal avenue to treat different categories of Meckel's cave and cavernous sinus neoplasms persist.

With unceasing energy to cultivate minimally invasive neurosurgical approaches, the endoscopic endonasal route has been tested, and the approach effectively performed, to provide a valid surgical window to these areas. In this dynamic and challenging scenario, another ventral endoscopic minimally invasive route-that is, the superior eyelid endoscopic transorbital approach —has been very recently proposed, and used in selected cases, to access the cavernous sinus and Meckel's cave regions.

METHODS The authors report the technical nuances of a combined and simultaneous endoscopic endonasal and transorbital surgical treatment of a patient with a Meckel's cave schwannoma. The operation involved collaboration among neurosurgery, otorhinolaryngology, and ophthalmology (oculoplastic surgery). The patient recovered well, had no neurological deficits, and was discharged to home 3 days after surgery.

RESULTS The multiportal combined route was proposed for the following reasons. The endonasal approach, considered to be more familiar to our skull base team, could allow control of possible damage of the internal carotid artery. From the endonasal perspective, the most inferior and medial portion of the tumor could be properly managed. Finally, the transorbital route, by means of opening the lateral wall of the cavernous sinus via the meningoorbital band, could allow control of the superolateral part of the tumor and, most importantly, could permit removal of the portion entering the posterior cranial fossa via the trigeminal pore. Simultaneous surgery with two surgical teams working together was planned in order to reduce operative time, hospital stay, and patient stress and discomfort, and to ensure "one-shot" complete tumor removal, with minimal or no complications.

CONCLUSIONS This study represents the translation into the real surgical setting of recent anatomical contributions related to the novel endoscopic transorbital approach and its simultaneous integration with the endoscopic endonasal pathway. Accordingly, it may pave the way for future applications related to minimally invasive, multiportal endoscopic surgery for skull base tumors.

https://thejns.org/doi/abs/10.3171/2020.4.JNS20707

KEYWORDS endoscopic endonasal; endoscopic transorbital; endoscopic skull base surgery; multiportal approach; trigeminal schwannoma; multiportal endoscopic; cavernous sinus; Meckel's cave; pituitary surgery; surgical technique

ABBREVIATIONS ENT = ear, nose, and throat; GTR = gross-total resection; ICA = internal carotid artery; NTR = near-total resection.

SUBMITTED March 15, 2020. ACCEPTED April 20, 2020.

INCLUDE WHEN CITING Published online July 10, 2020; DOI: 10.3171/2020.4.JNS20707. 
$\mathrm{T}$ Hroughout the years, Meckel's cave and cavernous sinus pathologies have been judged to be off-limits because of the high rates of morbidity, like cranial nerve injury and/or massive bleedings. ${ }^{1-3}$ During the past 2 decades, several studies have defined various surgical routes with tolerable morbidity and mortality rates. ${ }^{4-12}$ Nevertheless, controversy related to the optimal avenue to treat different categories of cavernous sinus neoplasms persists, and the cavernous sinus area is thought to be one of the most challenging spots for many neurosurgeons.

With unceasing energy to cultivate minimally invasive neurosurgical approaches, the endoscopic endonasal route has been tested, and the approach effectively performed, to provide a valid surgical window to the cavernous sinus. ${ }^{11,13-18}$

In this dynamic and challenging scenario, another ventral endoscopic minimally invasive route - that is, the superior eyelid endoscopic transorbital approach-has been very recently proposed, and used in selected cases, to access the middle cranial fossa and cavernous sinus regions. ${ }^{19-22}$ Used over the years for orbital surgeons to perform orbital surgery, the deep lateral orbital wall approach has also been proposed as a possible way to access not only the orbital apex but also the cavernous sinus. ${ }^{23}$

With the general aim of translating anatomical results $^{24-27}$ into real clinical and surgical contexts, we describe the technical nuances of a combined and simultaneous multiportal endoscopic endonasal and transorbital surgery for a cavernous sinus schwannoma. The multiportal combined approach was planned for the following intentions. The endonasal approach, more familiar to our skull base team, could permit control of possible injury of the internal carotid artery (ICA). From the endonasal perspective, the most inferior and medial portion of the tumor could be properly managed. Finally, the transorbital route, by means of interdural exposure of the cavernous sinus lateral wall, could allow control of the superolateral part of the tumor and, most importantly, could permit removal of the portion entering the posterior cranial fossa via the trigeminal pore.

It has to be stressed that simultaneous surgery using two surgical teams working together was planned in order to reduce surgical time, hospital stay, and patient stress and discomfort, and to guarantee complete tumor removal.

\section{Case Report \\ Preoperative Details}

A 43-year-old man was referred to our center for a second opinion regarding a left cavernous sinus lesion. The patient presented with a few months' history of paresthesia in the mandibular and maxillary branch dermatomes of the left trigeminal nerve, with occasional neuralgia at the level of the ipsilateral ophthalmic branch dermatome. MRI showed a 2.5-cm lesion, with enhancement after gadolinium injection, located in the left cavernous sinus, displacing the temporal lobe laterally and sharing an intimate relationship with the ICA in its most medial aspect (Fig. 1). Radiotherapy was offered as a first option at the patient's reference hospital. After considering many factors, such as the possible benign nature of the lesion, the experience of the surgical team in endoscopic skull base surgery, and the young age of the patient, endoscopic endonasal and transorbital combined and simultaneous surgery was offered (Fig. 2). After careful consideration and explanation of the pros and cons of the surgical treatment, including the surgery's risks and benefits, informed consent was signed by the patient and the surgical procedure was planned. Approval from the ethics committee was obtained.

\section{Surgical Procedure}

The operation involved collaboration among the neurosurgery, ENT (ear, nose, throat), and ophthalmology teams. One of the main concerns was the spatial organization of each surgeon in the surgical theater. In order to fix the space offered by the operating room, and with the aim of ensuring the comfort of all surgeons, dedicated planning was performed before surgery. Accordingly, after induction of general anesthesia, with the patient placed supine and with his head fixed with a Mayfield head holder, two surgeons were placed on the right side of the patient, while the other two stood on the left side. Specifically, an ENT surgeon and a neurosurgeon stood on the right side, while an oculoplastic surgeon and a neurosurgeon stood on the left side (tumor side). Endoscopic monitors were placed in front of each surgical team, while the neuronavigation monitor was on the most lateral right side (Fig. 3).

Hence, the surgical procedure started simultaneously in the nasal and left orbital cavities accordingly. Full face preparation with $10 \%$ povidone-iodine solution was performed; it is important to avoid spilling of the solution into the eyes, as this might cause an irritation of the ocular surface. After instilling two drops of topical anesthesia (oxybuprocaine hydrochloride $4 \mathrm{mg} / \mathrm{ml}$ and tetracaine hydrochloride $1 \mathrm{mg} / \mathrm{ml}$ ) and a small amount of carbomer ophthalmic gel $2 \mathrm{mg} / \mathrm{g}$ into the left inferior conjunctival fornix, a corneal protector was placed on the ipsilateral side to avoid potential corneal damage. For the endoscopic endonasal route, a transpterygoid approach to the medial wall of the cavernous sinus was accomplished, according to the technique already presented in the literature. ${ }^{28-30}$ Briefly, a left middle turbinectomy and ethmoidectomy were completed, followed by a wide sphenoidotomy. The medial portion of the posterior wall of the maxillary sinus was therefore resected to expose the posterior wall of the maxillary antrum and the vertical process of the palatine bone that was drilled out, together with exposure of the medial pterygoid process. Hence, the medial wall of the cavernous sinus was reached. On the other hand, for the endoscopic transorbital route, an interdural approach to the cavernous sinus, with dissection of the meningoorbital band, was performed, as previously described in the literature. ${ }^{26}$ The upper eyelid skin crease was identified and marked with a surgical marker pen, and the line was extended inferolaterally to the external aspect of the lateral orbital rim. After infiltration of $0.5 \%$ bupivacaine with 1:200,000 epinephrine under the eyelid skin and deep in the anterior area of the temporal fossa, a skinonly incision was made using a Colorado microdissection needle. The orbicularis oculi muscle was also spared with the monopolar electrocautery to the level of the orbital septum. At this point, it is important not to damage the 

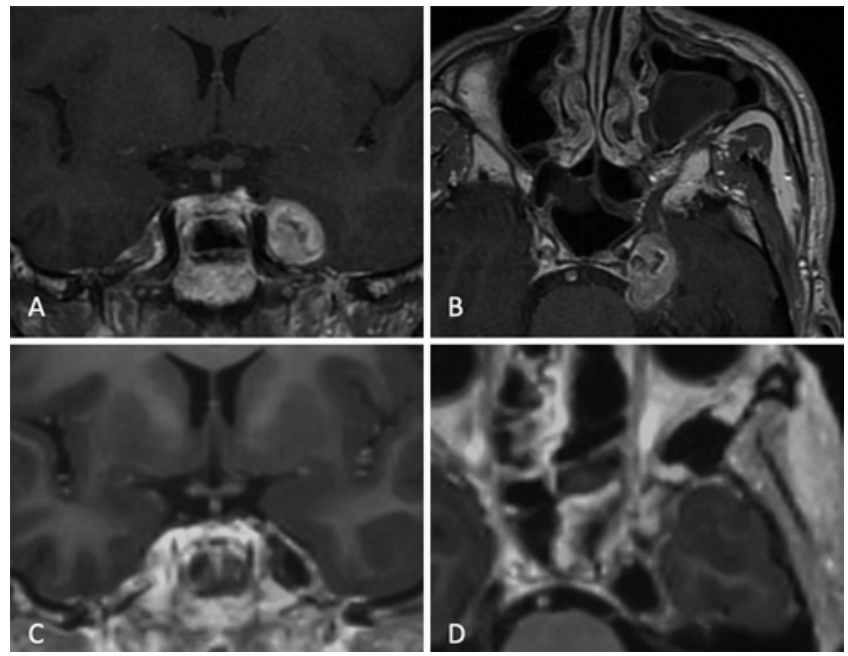

FIG. 1. Preoperative coronal (A) and axial (B) MR images showing a contrast-enhancing lesion located in the parasellar region at the level of the left Meckel's cave. Postoperative coronal (C) and axial (D) MR images demonstrating total tumor removal.

orbital septum and continue the dissection in the preseptal plane. The skin-muscle flap was raised superolaterally until the superolateral periosteum of the orbital rim was identified and exposed widely; the periosteum was then incised using the Colorado needle $1 \mathrm{~cm}$ posterior to the orbital rim and elevated using a Freer periosteal elevator to gain access to the bony orbital rim. Combining the periosteal elevator and different sizes of brain retractors, the periosteum was dissected off the orbital lateral wall. The zygomaticofacial and zygomaticotemporal bundles were identified, coagulated using a bipolar cautery, and cut using Stevens scissors. From this point to the end of the procedure, the orbital contents, covered by the periorbit, were displaced medially and protected with a brain retractor, and the endoscope was inserted into the field.

An ab interno orbital lateral wall decompression was performed, starting on the inner side of the orbital rim using a cutting 5-mm burr power drill and progressing posteriorly; once the bone marrow of the greater sphenoid wing was exposed, a 5-mm diamond burr was preferred so as to continue with a more controlled drilling. The limits of the drilling were, anteriorly, $1 \mathrm{~cm}$ posterior to the lateral orbital rim; superiorly, the "lacrimal keyhole" area; superoposteriorly, the superior orbital fissure; and inferiorly, the inferior orbital fissure. Extensive drilling of the greater sphenoid wing and lesser sphenoid wing was performed until the middle cranial fossa dura was reached. With minimal modifications, a similar technique is commonly used by oculoplastic surgeons to decompress the deep lateral orbital wall in thyroid eye disease and has been described previously. ${ }^{31}$ Afterwards, opening of the interdural space of the cavernous sinus was achieved by means of dissecting the meningoorbital band, as previously described..$^{21,24}$

Image guidance was used during the surgical procedure. More specifically, image guidance was useful during the peeling off of the lateral wall of the cavernous sinus, since we wanted to be sure to get the target via the trans- orbital route. Moreover, neuronavigation was helpful during the bone opening at the level of the lateral wall of the sphenoid sinus via the endonasal approach.

After the approach was completed from each corridor (endonasal and transorbital), both sides of the tumor were exposed, i.e., the most inferomedial aspect from the endonasal corridor and the most posterior and lateral side from the transorbital route (Fig. 4). Tumor removal was accomplished via both corridors by means of curettage, suction, and ultrasonic aspiration. Adequate hemostasis was ensured after tumor removal (Fig. 5).

In cases of intraoperative ICA rupture, for efficient control of bleeding while maintaining a patent ICA, our standard technique is to pack the site of the rupture with a muscle graft, according to data presented in the main literature. ${ }^{32,33}$ In this case, packing would have been performed via the endonasal opening at the level of the cavernous sinus medial wall. The procedure will then be followed by endovascular treatment, according to standard practices. ${ }^{34}$ Another possible option would be the insertion of a Fogarty balloon catheter in order to achieve further compression. ${ }^{35}$

Proper reconstruction of the skull base defect was achieved for both corridors. In particular, a nasoseptal flap harvested from the ipsilateral side was used to cover, together with dural substitute, the osteodural defect from the endonasal perspective; on the other hand, autologous fat graft and dural substitute were used to reconstruct the transorbital window. The orbital approach was closed in different layers, using four single stitches of 5-0 VICRYL to close the periosteum, three 5-0 VICRYL stitches to the orbicularis in the lateral canthus, and a running 6-0 PROLENE suture to close the skin (Video 1).

VIDEO 1. In this video we show a multiportal endoscopic approach for a left Meckel's cave schwannoma. Preoperative coronal MR images illustrate in green the lesion area amenable for the endonasal route, while in yellow is the other segment of the tumor that can be reached via the transorbital pathway. The operation involves a multidisciplinary skull base team. With the patient placed supine, two surgeons, working in the endonasal corridor, stand on the right side, while the other two surgeons, working in the transorbital corridor, stand on the left side-that is, the tumor side. The surgical procedure starts simultaneously in both ports. The established three- to four-hands technique for endoscopic skull base surgery is used-that is, with the endoscope placed in the upper corner of the surgical field and two working instruments placed below. For the endoscopic endonasal port, which can be seen on the left side of the screen, a transpterygoid approach to the medial wall of the cavernous sinus is accomplished. On the other hand, for the endoscopic superior eyelid transorbital route, shown on the right side of the screen, a middle fossa approach is performed by means of drilling of the lateral orbital wall and greater sphenoid wing. Still images as well as 3D CT scans showing the main anatomical landmarks from both perspectives are provided. The bone opening is enlarged from both corridors, and still images with corresponding 3D CT scans are shown. Afterward, interdural dissection of the lateral wall of the cavernous sinus is achieved from the transorbital corridor. So, while from the endonasal perspective it is possible to manage the most inferomedial portion of the lesion, from the transorbital port its superolateral and posterior portions are exposed and progressively removed. Here we can see details of the removal of the tumor via the transorbital route. Instruments from the endonasal corridors are visible inside the surgical field. Progressive dissection of the most superolateral and posterior borders of the lesion is performed while traction is done by the endonasal team. Finally, total 

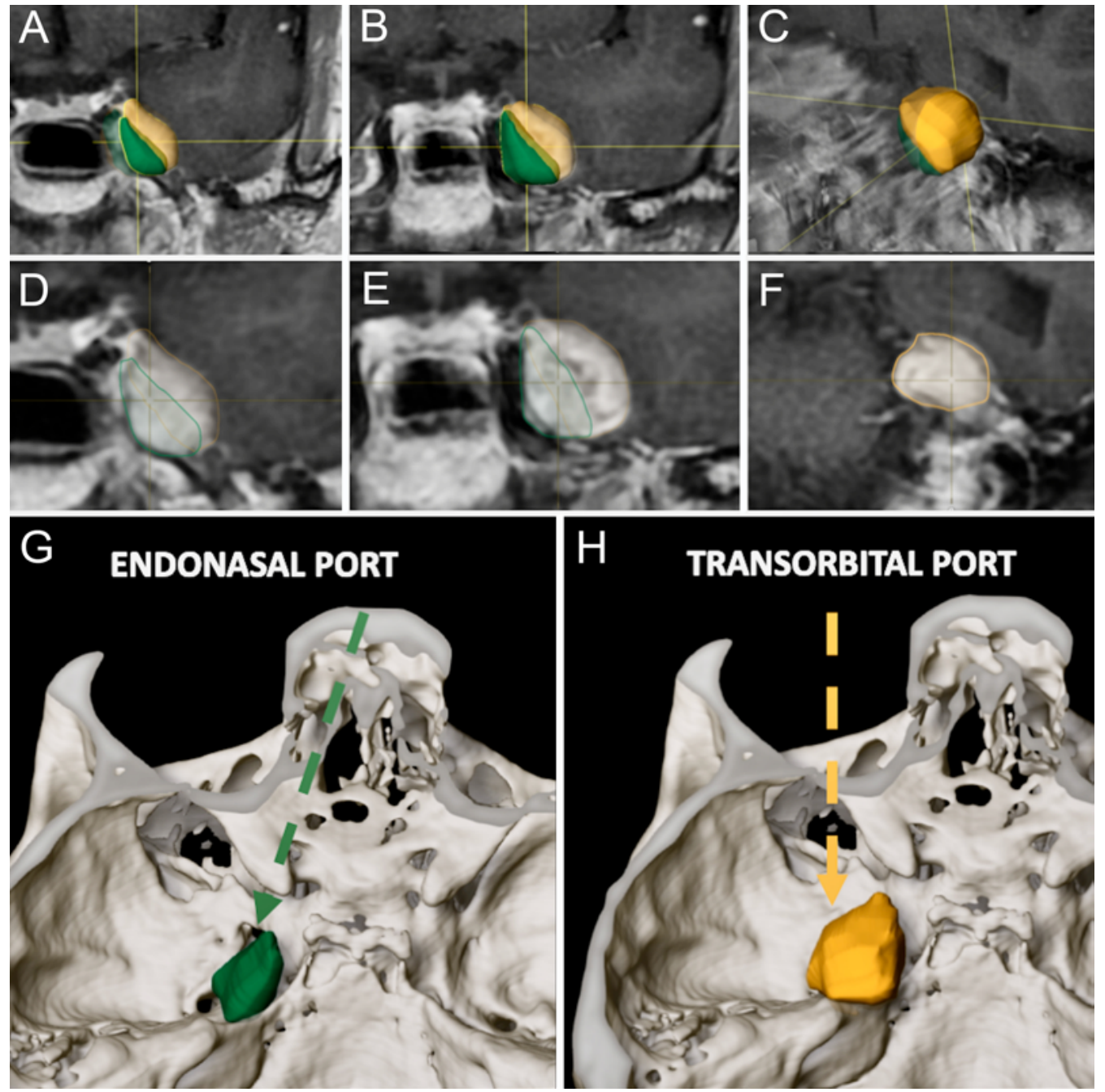

FIG. 2. A-C: Anteroposterior sequential preoperative coronal MR images showing in green the portion of the lesion possibly amenable to resection via the endonasal route and in yellow the other segment of the tumor that can be reached via the transorbital pathway. D-F: Three-dimensional reconstruction of the tumor has been performed using the same coronal slices and from anterior to posterior. $\mathbf{G}$ and $\mathbf{H}$ : The segmented 3D object representing the tumor has been fused with the CT scans of the skull base to provide a dedicated spatial orientation, thus giving the perspectives of the two complementary routes used in this case, i.e., endonasal/"nonparallel to the major tumor axis," represented by a green arrow (G) and transorbital/"parallel to the major tumor axis," represented with a yellow arrow $(\mathrm{H})$.

removal is completed. After obtaining adequate hemostasis, proper reconstruction of the skull base defect is done for both corridors. In particular, a nasoseptal flap together with a dural substitute is used to cover the osteodural defect from the endonasal perspective; on the other hand, an autologous fat graft and dural substitute are used to reconstruct the transorbital window. The orbital approach is closed in different layers. Postoperative MR images show complete tumor removal, and at last follow-up the patient was fine, with no neurological signs and satisfactory general and cosmetic outcomes. Copyright Alberto Di Somma. Published with permission. Click here to view.

The entire procedure lasted 4 hours.

\section{Pathology and Outcome}

The pathology report showed a schwannoma of the trigeminal nerve with diffuse and intense positivity for S-100 protein but with no histological signs of malignancy.
The patient recovered well after surgery, with minimal upper eyelid edema and minimal hypesthesia in the mandibular and maxillary branch dermatomes of the left trigeminal nerve, as well as slight diplopia in the lateral left gaze, all of which progressively resolved during the 1st week after surgery (Fig. 6). No neuralgia, cranial nerve deficit, CSF leak, or surgical wound complications were detected. CT scanning showed no complications and MRI indicated complete tumor removal. The patient was discharged home 3 days after surgery (Fig. 1). At the last follow-up visit, the patient was fine, with no trigeminal neuralgia, hypo-/paresthesia, or any other neurological signs. General health of the Medical Outcomes portion of the SF-36 was good (physical functioning 100\%, general health $85 \%$, and health change $100 \%$ [see Supplemental Data]), and satisfaction calculated using the Patient Satisfaction Survey related to cranial surgical approaches ${ }^{36}$ 


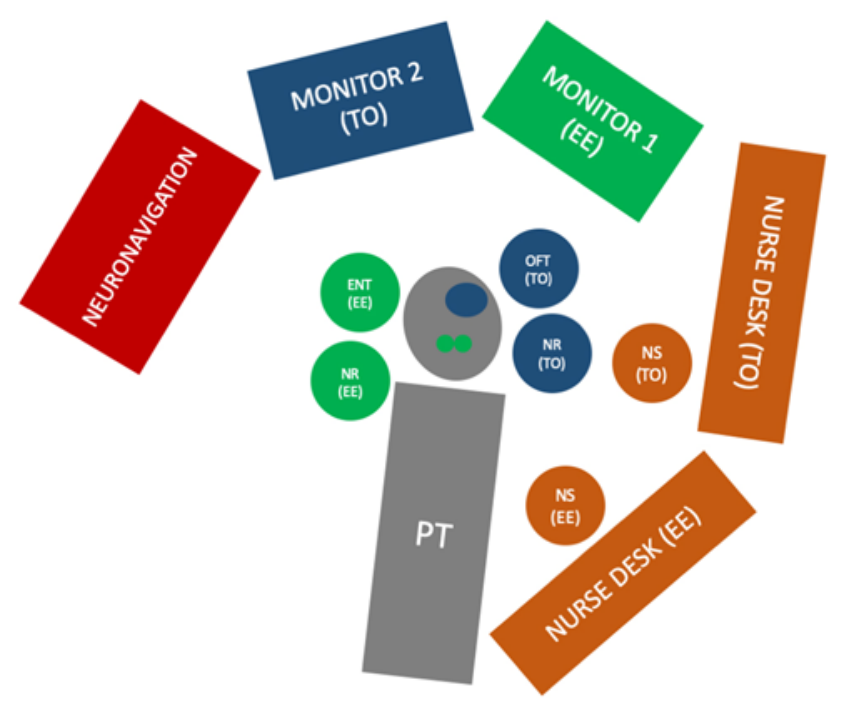

ANESTHESIA +/- NEUROMONITORING

FIG. 3. Spatial organization of the multidisciplinary team in the surgical theater. With the patient placed supine, two surgeons, working in the endonasal corridor, stand on the right side, and the other two surgeons, working in the transorbital corridor, stand on the left side, which is the side of the tumor. EE = endoscopic endonasal; ENT = otorhinolaryngology surgeon; NR = neurosurgeon; NS = nurse; OFT = oculoplastic surgeon; $\mathrm{PT}$ = patient; $\mathrm{TO}=$ transorbital. Figure is available in color online only.

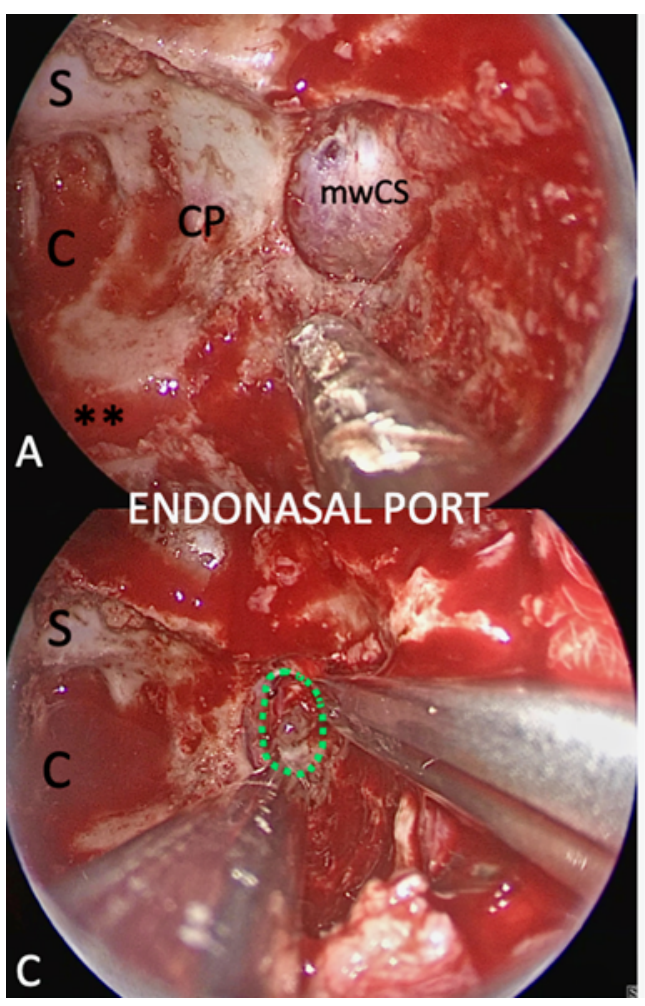

resulted in a satisfactory score (0 in sensory symptoms, cosmetic complaints, palpable cranial irregularities; 1 in craniotomy-related pain; and 3 in patient satisfaction).

\section{Discussion}

Skull base lesions encompassing the middle cranial fossa, including the cavernous sinus, and Meckel's cave still pose a unique surgical challenge to neurosurgeons due to the anatomical confluence of surrounding critical neurovascular structures in several deep and close compartments.

Conventional approaches, both extra- and intradural, have been performed based on the specific localization and features of the neoplasm and its extension involving adjacent neurovascular structures. All of the cranial routes, including frontotemporal and middle fossa approaches, as well as a subtemporal extradural or anterior transpetrosal approach, with or without modifications in bony removal, have been well performed over the years.

In the last 2 decades, the advancement and development of minimally invasive techniques in endoscopic skull base surgery have superseded conventional transcranial approaches due to the finest visualization of the operative field and to the lack of or a minimal skin incision and, therefore, absence of temporal muscle atrophy and brain retraction.

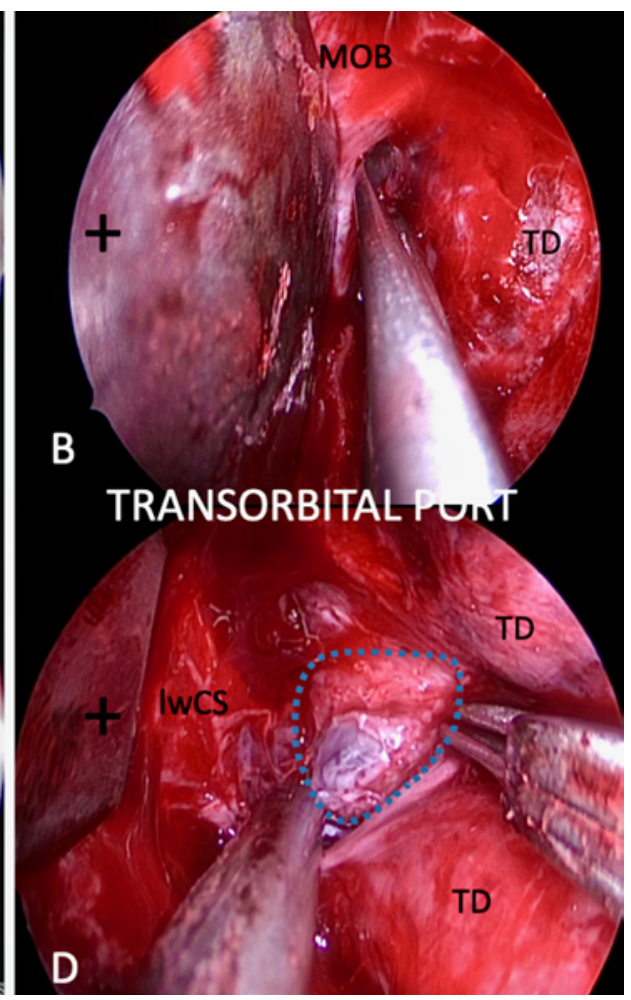

FIG. 4. Combined and simultaneous endoscopic views via the endonasal route (A, right side of the picture) and transorbital corridor ( $\mathbf{B}$, left side of the picture). The tumor is reached via both corridors, thus permitting proper management of its medial $(\mathbf{C}$, dotted green line, endonasal port) and lateral ( $\mathbf{D}$, dotted blue line, transorbital port) parts. ${ }^{*}$ Floor of the sphenoid sinus; dotted green line shows the margin of the tumor reached via the endonasal corridor; dotted blue line shows the margin of the tumor reached via the transorbital corridor. $\mathrm{C}=$ clivus; $\mathrm{CP}=$ carotid protuberance; IWCS = lateral wall of the cavernous sinus; $\mathrm{MOB}=$ meningoorbital band; mwCS = medial wall of the cavernous sinus; $S=$ sella; $T D=$ temporal dura. Figure is available in color online only. 

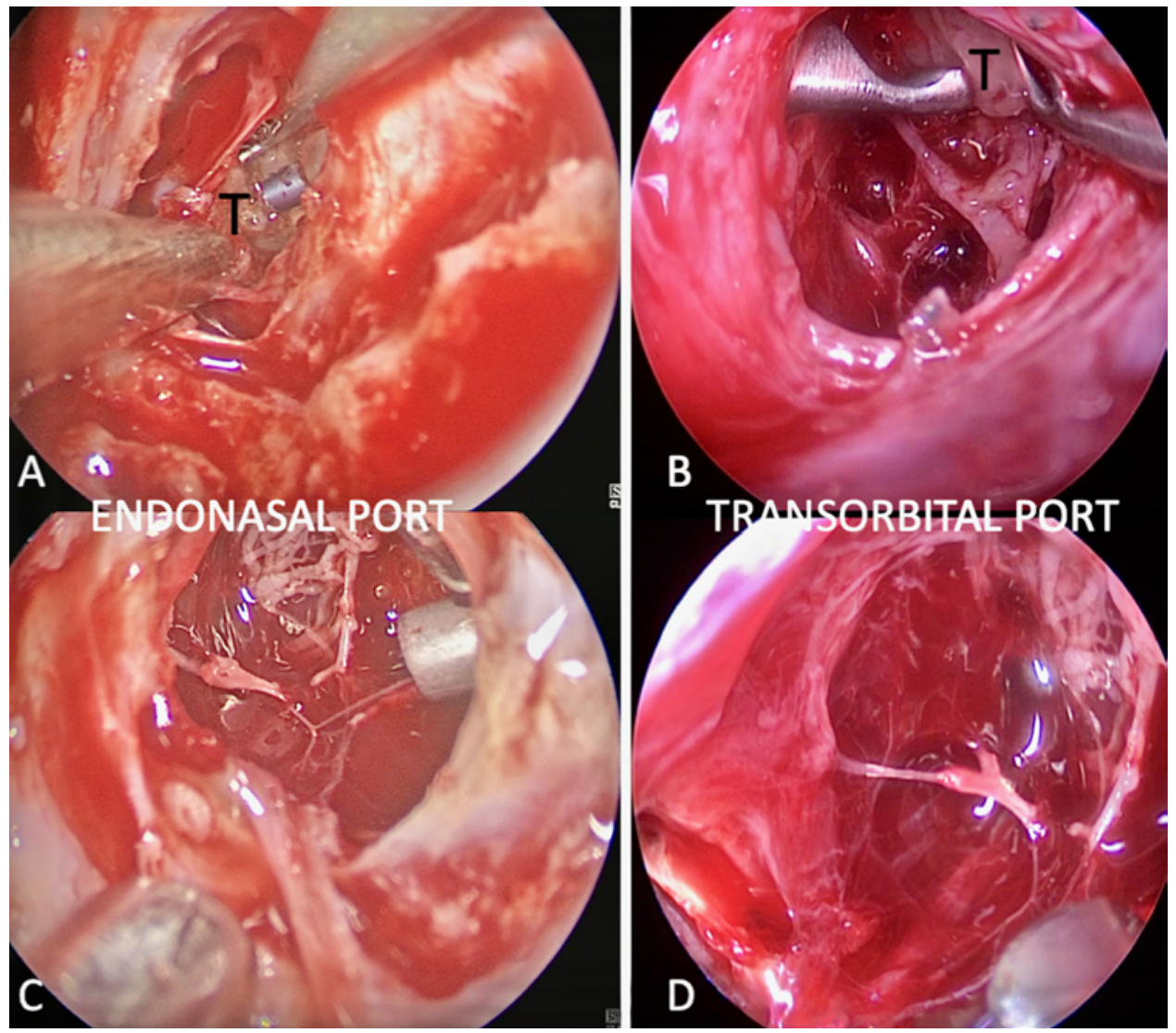

FIG. 5. Tumor (T) removal via both combined and simultaneous multiportal endoscopic pathways (endonasal [A]; transorbital [B]). Surgical cavity after complete tumor removal (C and D). Figure is available in color online only.

In this context, the recently introduced endoscopic transorbital approach, performed via the superior eyelid crease, offers a direct and practical way to reach the lateral and superolateral orbital wall to target middle and anterior fossa lesions. This novel surgical corridor provides the
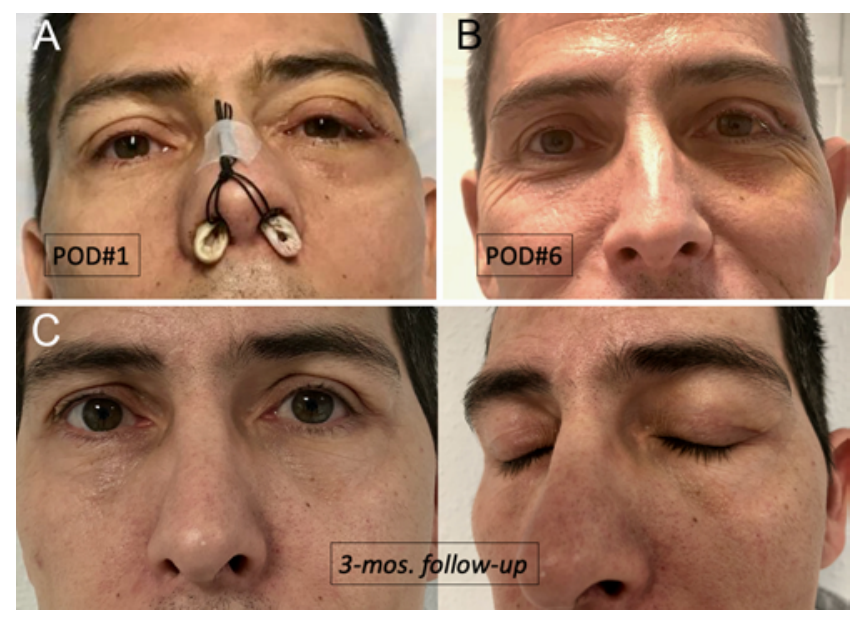

FIG. 6. Cosmetic results at 1 (A) and 6 (B) days after surgery and at the 3-month follow-up visit (C). No visible scar can be seen with the patient's eye opened. $\mathrm{POD}=$ postoperative day. Figure is available in color online only. most straightforward route to Meckel's cave and the lateral wall of the cavernous sinus with minimal morbidity associated with mild eyelid edema and scarring. However, such a transorbital ventral route may not allow adequate exposure and control of the most medial and inferior aspect of the tumor; further, possible damage of the ICA may be difficult to manage. Currently, a combined multiportal transorbital endonasal endoscopic approach has been described as a safer and more reliable approach to access complex skull base lesions. ${ }^{37-41}$

Hence, with such a background, in this paper we described some technical nuances of a combined and simultaneous endoscopic endonasal and transorbital surgery for a cavernous sinus schwannoma. Both approaches offer access to Meckel's cave without disruption of the temporalis muscle or the need for an external craniotomy.

The primary advantages of the transorbital approach include the lack of relevant brain retraction, less scar tissue formation along the eyelid crease, and no disruption or consequent postoperative atrophy of the temporalis muscle. The transorbital pathway also allows interdural dissection to enter the cavernous sinus using a peeling technique and facilitates relatively easy manipulation of a tumor at its lateral and posterior borders. In addition, a minimal scar hidden within the natural eyelid crease contributes to an improved cosmetic effect. The primary disadvantage of the transorbital approach is the unfamiliarity of most sur- 
TABLE 1. Main factors addressing the advantages and limitations of the combined endoscopic endonasal and transorbital approach to Meckel's cave

\begin{tabular}{lc}
\hline \multicolumn{1}{c}{ Advantages } & Limitations \\
\hline Endoscopic endonasal & Endoscopic endonasal \\
\hline Control of the ICA at the lacerum \& cavernous segments & Difficult to control posterior fossa portion of the tumor \\
\hline Exposure of the medial MC & Difficulties if conchal sphenoid sinus \\
\hline Exposure \& removal of the medial \& inferior portion of the lesion & $\begin{array}{c}\text { Difficulty controlling the lateral \& superior portion ("nonparallel" route } \\
\text { to the major axis of the tumor) }\end{array}$ \\
\hline Experience of the skull base team & Endoscopic transorbital \\
\hline Endoscopic transorbital & Difficulty controlling the most medial portion of the lesion \\
\hline "Parallel" route to the major axis of the lesion in MC & Unfamiliarity w/ the approach \\
\hline Exposure of the vertical portion of the greater sphenoid wing \& MCF floor & \\
\hline Interdural exposure of the lateral wall of the cavernous sinus & \\
\hline Removal of the lateral portion of the lesion & \\
\hline Removal of the posterior fossa portion of the lesion & Combined \& simultaneous surgery \\
\hline Combined \& simultaneous surgery & Spatial organization of the operating room \\
\hline $\begin{array}{l}\text { Check for complete tumor removal from different \& complementary } \\
\text { perspectives }\end{array}$ & Reproducibility \\
\hline Control of hidden angles of one route via the other route & Take into account possible complications of the 2 approaches \\
\hline Minimize patient stress \& discomfort & $\begin{array}{l}\text { More than 2 surgical specialties involved (ENT, neurosurgery, oculo- } \\
\text { plastic surgery) }\end{array}$ \\
\hline Take into account advantages of both approaches & \\
\hline
\end{tabular}

$\mathrm{MC}=$ Meckel's cave; $\mathrm{MCF}=$ middle cranial fossa .

geons with the orbital anatomy from a ventral viewpoint. Orbital surgery is always challenging due to its anatomical complexity and the presence of important structures in a very small area, such as the extraocular muscles; the lacrimal gland; several cranial nerves, including the optic nerve; and the eyeball. Complications derived from orbital manipulation can run the gamut-from less severe complications, such as skin scar formation at the access point or upper eyelid ptosis that can usually be repaired; to diplopia secondary to muscle or nerve damage, or dry eye due to lacrimal gland impairment; to the worst complications, such as severe visual deterioration or even blindness.

The endoscopic endonasal surgical anatomy is more familiar than that of the transorbital approach to access the cavernous sinus. Approaching the lateral and posterior margins of a tumor remains a challenge from the endonasal perspective. However, the endonasal route can be an effective pathway to control the ICA from the lacerum to the cavernous segment and to give confidence to the surgical team at the beginning of the learning curve by giving them another minimally invasive skull base approach, i.e., the transorbital approach.

In this study, we highlighted some technical nuances related to simultaneous and combined endonasal and transorbital surgery.

Some advantages of simultaneous and combined surgery are, among others, comprehensive control of the tumor margins and control of the hidden angle of one route with the other approach (Table 1). Indeed, if on one side the transorbital route permits us to manage the most superolateral and posterior portion of the lesion by means of following its major axis ("parallel" route to the tumor), on the other side the most inferomedial portion of the lesion is reached via the endonasal path in an "nonparallel" manner (nonparallel route to the tumor). In these terms, conceptually, this multiportal approach shares some similarities with the laparoscopic surgery in which, by means of inserting trocars in the abdominal cavity from various angles, comprehensive visualization and management of the target area can be achieved. The use of multiple ports to access the skull base may empower advanced manipulation, provide direct visual feedback, and expand the indications related to complex neoplasms of the skull base, in selected cases. Furthermore, it has been shown that the intersection of more than one trajectory at the target forms a cavity, which creates additional space for manipulation around the target region of interest. ${ }^{42}$

Further details need to be discussed regarding the surgical management of trigeminal schwannomas via an endoscopic transorbital or endoscopic endonasal approach.

In a recent pure transorbital series, Jeon et al. ${ }^{21}$ reported that gross-total resection (GTR) was performed in 7 of the 9 patients $(77.8 \%)$, and if only trigeminal schwannomas were examined, it would be possible to highlight that in 3 of the 4 patients (75\%), GTR was achieved. However, in the study by Kong and colleagues, ${ }^{22}$ the one non-GTR case featured a unique complexity and volume. Similar results were reported in another paper by the same group. ${ }^{20}$ In another series focusing on 25 cases of trigeminal schwannomas, Park et al. ${ }^{43}$ demonstrated that GTR and near-total resection (NTR) were achieved in $76 \%$ of the cases. The GTR/NTR rates of endoscopic transorbital approach related to trigeminal schwannomas extending to both the middle and posterior cranial fossae were $75 \%$. 
On the other hand, analyzing the most recent endoscopic endonasal series, the GTR rate for trigeminal schwannomas varies from $33 \%$ to $80 \%,{ }^{44-47}$ with some of the non-GTR patients presenting with huge volumes and a complexity that made these cases very different from the one presented in this article. ${ }^{44-47}$ Furthermore, it should be stressed that, in one study describing the endoscopic endonasal management of trigeminal schwannomas extending into the infratemporal fossa, $100 \%$ removal was reported. ${ }^{48}$ In another recent article, ${ }^{49}$ GTR via the endoscopic endonasal route was achieved in all patients; in that study one patient had previously undergone a transcranial approach for a subtotal resection at the same author's institution (i.e., at Dr. Wu's institution). ${ }^{49}$

Hence, given that in the majority of studies with a single pathway GTR rates account for $75 \%-80 \%$ of the cases, we thought that by combining the two complementary routes (endonasal/"nonparallel to the major tumor axis" and transorbital/"parallel to the major tumor axis"), taking advantage of the endoscopic skull base technique, a complete tumor removal could be planned and achieved in the present case. Thus, we planned to manage the most inferomedial portion of the lesion from the endonasal perspective and from the transorbital approach, the tumor's superolateral and posterior portions.

Reviewing the literature concerning the multiportal combined endonasal and transorbital approach to the skull base, we found a total of 24 patients (16 women, 8 men; mean age 32.5 years) with skull base neoplasms who underwent a combined endoscopic endonasal and transorbital approach. ${ }^{20-22,37,50,51}$ The literature review revealed that in the majority of cases the diagnosis was a meningioma $(15 / 24$ cases, $63 \%)$, followed by chondrosarcoma (2/24 cases, $8 \%$ ). Interestingly, in $29 \%$ of the cases, such a combined approach was used to treat a recurrent neoplasm. Regarding the outcome, a GTR was achieved in $42 \%$ of cases, subtotal removal in 50\% of cases, and NTR in $8 \%$ of cases. The most frequently reported complications were eyelid edema, trigeminal hypesthesia, diplopia, and upper eyelid ptosis.

It should be stressed that, for lesions located on the right side, a slightly different organizational setup should be used. In fact, in the case of a tumor located on the right side of the patient, 3 surgeons-2 working in the transorbital corridor and 1 in the endonasal corridor-stand on the right side, while a fourth surgeon-working in the endonasal corridor-stands on the left side.

Finally, it has to be mentioned that interdisciplinary cooperation among neurosurgeons, ENT surgeons, ophthalmologists, and anesthesiologists is mandatory in order to achieve satisfactory results in such a complex endoscopic minimally invasive surgery. Further cases are needed to prove the efficacy and safety of such combined multiportal approaches to a complex skull base target.

\section{Study Limitations}

This study has limitations that need to be considered. One of the main limitations is its single-case nature. According to the classes of evidence in neurosurgery, ${ }^{52}$ our study can be categorized as "evidence level V."

\section{Conclusions}

We have described a multiportal (endoscopic endonasal and endoscopic transorbital) approach to Meckel's cave for the resection of a trigeminal schwannoma. In the past few years, we have seen several reports on the anatomical bases for these approaches. Detailed technical nuances have been provided, including the spatial arrangement of the surgical teams in the operative theater. This case report represents the translation into a real surgical setting of recent anatomical contributions related to the novel endoscopic transorbital approach and its simultaneous integration with the endoscopic endonasal pathway.

Of course, questions will linger about the procedure's safety, reproducibility, and particularly complications. Those can only be answered through the disclosure of detailed series including cases like the one presented here. Accordingly, the case presented herein may pave the way for future applications related to minimally invasive endoscopic surgery for skull base tumors.

\section{Acknowledgments}

This project was partially supported by grants from the "Instituto de Salud Carlos III" (PI19/00592) and the "Fundacio La Marato de TV3" (Reg. 95/210; Codi projecte: 201914).

\section{References}

1. Akutsu H, Kreutzer J, Fahlbusch R, Buchfelder M. Transsphenoidal decompression of the sellar floor for cavernous sinus meningiomas: experience with 21 patients. Neurosurgery. 2009;65(1):54-62.

2. Cusimano MD, Sekhar LN, Sen CN, et al. The results of surgery for benign tumors of the cavernous sinus. Neurosurgery. 1995;37(1):1-10.

3. O'Sullivan MG, van Loveren HR, Tew JM Jr. The surgical resectability of meningiomas of the cavernous sinus. Neurosurgery. 1997;40(2):238-247.

4. Ferreli F, Turri-Zanoni M, Canevari FR, et al. Endoscopic endonasal management of non-functioning pituitary adenomas with cavernous sinus invasion: a 10- year experience. Rhinology. 2015;53(4):308-316.

5. Sekhar LN, Sen CN, Jho HD, Janecka IP. Surgical treatment of intracavernous neoplasms: a four-year experience. Neurosurgery. 1989;24(1):18-30.

6. Knosp E, Perneczky A, Koos WT, et al. Meningiomas of the space of the cavernous sinus. Neurosurgery. 1996;38(3):434444.

7. Al-Mefty O. Supraorbital-pterional approach to skull base lesions. Neurosurgery. 1987;21(4):474-477.

8. Hakuba A, Nishimura S, Shirakata S, Tsukamoto M. Surgical approaches to the cavernous sinus. Report of 19 cases (author's transl). Article in Japanese. Neurol Med Chir (Tokyo). 1982;22(4):295-308.

9. Hakuba A, Tanaka K, Suzuki T, Nishimura S. A combined orbitozygomatic infratemporal epidural and subdural approach for lesions involving the entire cavernous sinus. $J$ Neurosurg. 1989;71(5 pt 1):699-704.

10. al-Mefty O, Anand VK. Zygomatic approach to skull-base lesions. J Neurosurg. 1990;73(5):668-673.

11. Frank G, Pasquini E. Endoscopic endonasal cavernous sinus surgery, with special reference to pituitary adenomas. Front Horm Res. 2006;34:64-82.

12. Perneczky A, Knosp E, Matula C. Cavernous sinus surgery. Approach through the lateral wall. Acta Neurochir (Wien). 1988;92(1-4):76-82. 
13. Frank G, Sciarretta V, Calbucci F, et al. The endoscopic transnasal transsphenoidal approach for the treatment of cranial base chordomas and chondrosarcomas. Neurosurgery. 2006;59(1)(suppl 1):ONS50-ONS57.

14. Frank G, Pasquini E. Endoscopic endonasal approaches to the cavernous sinus: surgical approaches. Neurosurgery. 2002;50(3):675

15. Zoli M, Milanese L, Bonfatti R, et al. Cavernous sinus invasion by pituitary adenomas: role of endoscopic endonasal surgery. J Neurosurg Sci. 2016;60(4):485-494.

16. Patrona A, Patel KS, Bander ED, et al. Endoscopic endonasal surgery for nonadenomatous, nonmeningeal pathology involving the cavernous sinus. J Neurosurg. 2017;126(3):880888.

17. Wei W, Qiuhang Z, Hongchuan G, Zhenlin W. Endoscopic endonasal biopsy for a tumor at the cavernous sinus and pons. J Craniofac Surg. 2015;26(2):e90-e92.

18. Jacquesson T, Berhouma M, Picart T, Jouanneau E. Total removal of a trigeminal schwannoma via the expanded endoscopic endonasal approach. Technical note. Acta Neurochir (Wien). 2015;157(6):935-938.

19. Dallan I, Sellari-Franceschini S, Turri-Zanoni M, et al. Endoscopic transorbital superior eyelid approach for the management of selected spheno-orbital meningiomas: preliminary experience. Oper Neurosurg (Hagerstown). 2018;14(3):243251

20. Lee MH, Hong SD, Woo KI, et al. Endoscopic endonasal versus transorbital surgery for middle cranial fossa tumors: comparison of clinical outcomes based on surgical corridors. World Neurosurg. 2019;122:e1491-e1504.

21. Jeon C, Hong CK, Woo KI, et al. Endoscopic transorbital surgery for Meckel's cave and middle cranial fossa tumors: surgical technique and early results. J Neurosurg. 2019;131(4):1126-1135.

22. Kong DS, Young SM, Hong CK, et al. Clinical and ophthalmological outcome of endoscopic transorbital surgery for cranioorbital tumors. J Neurosurg. 2018;131(3):667-675.

23. Goldberg RA, Shorr N, Arnold AC, Garcia GH. Deep transorbital approach to the apex and cavernous sinus. Ophthal Plast Reconstr Surg. 1998;14(5):336-341.

24. Di Somma A, Andaluz N, Cavallo LM, et al. Endoscopic transorbital superior eyelid approach: anatomical study from a neurosurgical perspective. J Neurosurg. 2018;129(5):12031216.

25. Di Somma A, Andaluz N, Cavallo LM, et al. Endoscopic transorbital route to the petrous apex: a feasibility anatomic study. Acta Neurochir (Wien). 2018;160(4):707-720.

26. Dallan I, Di Somma A, Prats-Galino A, et al. Endoscopic transorbital route to the cavernous sinus through the meningo-orbital band: a descriptive anatomical study. J Neurosurg. 2017;127(3):622-629.

27. Priddy BH, Nunes CF, Beer-Furlan A, et al. A side door to Meckel's cave: anatomic feasibility study for the lateral transorbital approach. Oper Neurosurg (Hagerstown). 2017;13(5):614-621.

28. de Lara D, Ditzel Filho LF, Prevedello DM, et al. Endonasal endoscopic approaches to the paramedian skull base. World Neurosurg. 2014;82(6)(suppl):S121-S129.

29. Fortes FS, Sennes LU, Carrau RL, et al. Endoscopic anatomy of the pterygopalatine fossa and the transpterygoid approach: development of a surgical instruction model. Laryngoscope. 2008;118(1):44-49.

30. Kaen A, Cárdenas Ruiz-Valdepeñas E, Di Somma A, et al. Refining the anatomic boundaries of the endoscopic endonasal transpterygoid approach: the "VELPPHA area" concept. $J$ Neurosurg. 2018;131(3):911-919.

31. Goldberg RA, Kim AJ, Kerivan KM. The lacrimal keyhole, orbital door jamb, and basin of the inferior orbital fissure. Three areas of deep bone in the lateral orbit. Arch Ophthalmol. 1998;116(12):1618-1624.
32. Wang WH, Lieber S, Lan MY, et al. Nasopharyngeal muscle patch for the management of internal carotid artery injury in endoscopic endonasal surgery. J Neurosurg. Published online October 18, 2019 doi:10.3171/2019.7.JNS191370

33. Gardner PA, Snyderman CH, Fernandez-Miranda JC, Jankowitz BT. Management of major vascular injury during endoscopic endonasal skull base surgery. Otolaryngol Clin North Am. 2016;49(3):819-828.

34. Zhang Y, Tian Z, Li C, et al. A modified endovascular treatment protocol for iatrogenic internal carotid artery injuries following endoscopic endonasal surgery. J Neurosurg. 2019;132(2):343-350.

35. Ruggeri A, Enseñat J, Prats-Galino A, et al. Endoscopic endonasal control of the paraclival internal carotid artery by Fogarty balloon catheter inflation: an anatomical study. $J$ Neurosurg. 2017;126(3):872-879.

36. Park J, Son W, Kwak Y, Ohk B. Pterional versus superciliary keyhole approach: direct comparison of approach-related complaints and satisfaction in the same patient. J Neurosurg. 2018;130(1):220-226.

37. Almeida JP, Omay SB, Shetty SR, et al. Transorbital endoscopic eyelid approach for resection of sphenoorbital meningiomas with predominant hyperostosis: report of 2 cases. $J$ Neurosurg. 2018;128(6):1885-1895.

38. Cavallo LM, Solari D, Cappabianca P. Multiportal approach to the skull base: one key, multiple gates. World Neurosurg. 2015;84(3):630-631

39. Ciporen JN, Moe KS, Ramanathan D, et al. Multiportal endoscopic approaches to the central skull base: a cadaveric study. World Neurosurg. 2010;73(6):705-712.

40. Dallan I, Castelnuovo P, Locatelli D, et al. Multiportal combined transorbital transnasal endoscopic approach for the management of selected skull base lesions: preliminary experience. World Neurosurg. 2015;84(1):97-107.

41. Tham T, Costantino P, Bruni M, et al. Multiportal combined transorbital and transnasal endoscopic resection of fibrous dysplasia. J Neurol Surg Rep. 2015;76(2):e291-e296.

42. Stenin I, Hansen S, Becker M, et al. Minimally invasive multiport surgery of the lateral skull base. BioMed Res Int. 2014;2014:379295

43. Park HH, Hong SD, Kim YH, et al. Endoscopic transorbital and endonasal approach for trigeminal schwannomas: a retrospective multicenter analysis (KOSEN-005). J Neurosurg. 2020;133(2):467-476

44. Raza SM, Donaldson AM, Mehta A, et al. Surgical management of trigeminal schwannomas: defining the role for endoscopic endonasal approaches. Neurosurg Focus. 2014;37(4):E17.

45. Shin SS, Gardner PA, Stefko ST, et al. Endoscopic endonasal approach for nonvestibular schwannomas. Neurosurgery. 2011;69(5):1046-1057.

46. Yang L, Hu L, Zhao W, et al. Endoscopic endonasal approach for trigeminal schwannomas: our experience of 39 patients in 10 years. Eur Arch Otorhinolaryngol. 2018;275(3):735-741.

47. Zoli M, Ratti S, Guaraldi F, et al. Endoscopic endonasal approach to primitive Meckel's cave tumors: a clinical series. Acta Neurochir (Wien). 2018;160(12):2349-2361.

48. Zhang Q, Feng K, Ge C, et al. Endoscopic endonasal management of trigeminal schwannomas extending into the infratemporal fossa. J Clin Neurosci. 2012;19(6):862-865.

49. Wu X, Xie SH, Tang B, et al. Single-stage endoscopic endonasal approach for the complete removal of trigeminal schwannomas occupying both the middle and posterior fossae. Neurosurg Rev. Published online February 19, 2020. doi:10.1007/s10143-020-01266-7

50. Janakiram TN, Bhatia Sharma S, Nahata Gattani V. Multiport combined endoscopic approach to nonembolized juvenile nasopharyngeal angiofibroma with parapharyngeal extension: an emerging concept. Int J Otolaryngol. 2016;2016:4203160. 
51. Ramakrishna R, Kim LJ, Bly RA, et al. Transorbital neuroendoscopic surgery for the treatment of skull base lesions. $J$ Clin Neurosci. 2016;24:99-104.

52. Rutka JT. Editorial. Classes of evidence in neurosurgery. $J$ Neurosurg. 2017;126(6):1747-1748.

\section{Disclosures}

Dr. Alobid reports being a consultant for Roche, Menarini, MSD, GSK, and Mylan. Dr. Di Somma reports being a consultant for Brainlab.

\section{Supplemental Information}

Videos

Video 1. https://vimeo.com/412270603.

Online-Only Content

Supplemental material is available with the online version of the article.
Supplemental Data. https://thejns.org/doi/suppl/10.3171/2020. 4.JNS20707.

\section{Author Contributions}

Conception and design: Di Somma, Enseñat. Acquisition of data: all authors. Analysis and interpretation of data: Di Somma, de Notaris, Enseñat. Drafting the article: Di Somma, de Notaris, Reyes, Enseñat. Critically revising the article: Di Somma, Langdon, de Notaris, Ortiz-Perez, Enseñat, Reyes. Reviewed submitted version of manuscript: all authors. Approved the final version of the manuscript on behalf of all authors: Di Somma. Study supervision: Di Somma, Alobid, Enseñat.

\section{Correspondence}

Alberto Di Somma: Institut Clínic de Neurociències (ICN), Hospital Clínic de Barcelona, Spain. adisomma@clinic.cat. 